\title{
Functional Analysis of the Mutations in the Human Cardiac $\beta$-Myosin that Are Responsible for Familial Hypertrophic Cardiomyopathy Implication for the Clinical Outcome
}

\author{
Masataka Sata and Mitsuo Ikebe \\ Department of Physiology, University of Massachusetts Medical Center, Worcester, Massachusetts 01655-0127
}

\begin{abstract}
More than 30 missense mutations in the $\beta$-cardiac myosin heavy chain gene have been shown to be responsible for familial hypertrophic cardiomyopathy. To clarify the effects of these point mutations on myosin motor function, we expressed wild-type and mutant human $\beta$-cardiac myosin heavy chains in insect cells with human cardiac light chains. The wild-type myosin was well purified with similar enzymatic and motor activities to those of the naturally isolated V3 cardiac myosin. $\mathrm{Arg}^{249} \rightarrow \mathrm{Gln}$ and $\mathrm{Arg}^{453} \rightarrow$ Cys mutations resulted in decreased actin translocating activity (61 and $23 \%$ of the wild-type, respectively) with decreased intrinsic ATPase activity. Arg $^{403} \rightarrow$ Gln mutation greatly decreased actin translocating activity ( $27 \%$ of wild type) with a 3.3 fold increased dissociation constant for actin, while intrinsic ATPase activity was unchanged. Val ${ }^{606} \rightarrow$ Met mutation only mildly affected the actin translocating activity as well as ATPase activity of myosin. The degree of deterioration by each mutation was closely correlated with the prognosis of the affected kindreds, indicating that myosin dysfunction caused by the point mutations is responsible for the pathogenesis of the disease. Structure/function relationship of myosin is discussed. (J. Clin. Invest. 1996. 98:2866-2873.) Key words: cardiomyopathy, hypertrophic - mutagenesis • myosin heavy chains $\bullet$ point mutation $\bullet$ genetics, medical
\end{abstract}

\section{Introduction}

Familial hypertrophic cardiomyopathy $(\mathrm{FHC})^{1}$ is an autosomal dominant disease characterized by a hypertrophied left ventricle in the absence of other causes of cardiac hypertrophy (1). While FHC is one of the causes of sudden and unexpected cardiac death, particularly in healthy young individuals, there is

Address correspondence to Mitsuo Ikebe, Department of Physiology, University of Massachusetts Medical Center, 55 Lake Avenue North, Worcester, MA 01655-0127. Phone: 508-856-6698; FAX: 508-8565997.

Received for publication 26 July 1996 and accepted in revised form 16 October 1996.

1. Abbreviations used in this paper: FHC, familial hypertrophic cardiomyopathy; MHC, myosin heavy chain; HMM, heavy meromyosin; LC1, light chain 1; LC2, light chain 2; S1, subfragment 1; S2, subfragment 2; RT-PCR, reverse transcriptase coupled polymerase chain reaction.

J. Clin. Invest.

(C) The American Society for Clinical Investigation, Inc. 0021-9738/96/12/2866/08 \$2.00

Volume 98, Number 12, December 1996, 2866-2873 no specific clinical feature which identifies the patients at risk of sudden death (2). At the present time, $>30$ different missense mutations in the $\beta$-cardiac myosin heavy chain (MHC) gene, which alter only a single amino acid residue, have been described (3-5). Phenotypic examinations of the genotypically defined kindreds showed that certain $\beta$-cardiac myosin heavy chain gene mutations carry greater risk of morbidity than others (6-9), demonstrating that mutations in $\beta$-cardiac $\mathrm{MHC}$ must be responsible for the pathogenesis of FHC. Since each of the altered amino acids has been highly conserved during evolution (6), it has been suggested that these residues are crucial for myosin function. However, it remained to be clarified how these myosin mutations affect myosin function, particularly its motor activity, thus causing cardiac malfunction.

One of the problems for physiological and/or biochemical studies of FHC is the difficulty of obtaining enough myosin sample from a patient's heart. To avoid this difficulty, Cuda et al. obtained biopsy samples from patient's slow skeletal muscle fibers which also express $\beta$-MHC along with skeletal muscle myosin and attempted to evaluate the effects of mutations on the $\beta$-myosin motor function (10). They selected $\beta$-myosin from the mixture of skeletal muscle myosin and $\beta$-myosin using a $\beta$-MHC specific antibody and showed that $\beta$-myosin from the patients translocated actin filaments much slower than normal control. However, their preparation is a mixture of the mutated and nonmutated $\beta$-myosins and it is postulated that the ratio of the normal and mutant $\beta$-myosins in patient's heart would be different from that in slow skeletal muscle fiber. Furthermore, since the data represent the activity of $\beta$-myosin of unknown ratio of the normal and mutant myosins, it is difficult to quantitatively evaluate the function of mutant $\beta$-myosin. Obviously, such an analysis can only be made with pure $\beta$-myosin mutant.

On the other hand, Sweeney et al. expressed rat $\alpha$-cardiac myosin in insect cells and reported that $\mathrm{Arg}^{403} \rightarrow$ Gln mutation resulted in reduction of actin activated ATPase activity and actin translocating activity (11). Problem is, however, only $\beta$-MHC but not $\alpha$-MHC is expressed in human adult left ventricle (12) and V1 cardiac myosin isoform, homodimer of $\alpha$-MHC, has three to fourfold higher activity than V3 isoform, homodimer of $\beta$-MHC, in both actin-translocating velocity and actin-activated ATPase activity (13-16). Divergence between $\alpha$ - and $\beta$-MHCs clusters in functionally important regions including ATP, actin, and light chain-binding sites (17). Therefore, the effect of the point mutation on the rat $\alpha$-cardiac myosin may be different from that on the human $\beta$-myosin.

The purpose of this study is to express functionally active recombinant human $\beta$-myosin mutants found in the FHC patients and to analyze the effects of each mutation on the motor activity. The active human $\beta$-MHCs expressed with human ventricular myosin light chains were successfully purified and the motor and enzymatic activities of myosin mutants were determined. Each $\beta$-MHC mutant showed abnormal function ac- 
cording to the site and the type of the mutation. Interestingly, the degree of deterioration by the mutation was well correlated with the mortality rate among the affected kindreds supporting the idea that the alteration of $\beta$-myosin motor activity by mutation is the critical factor for the prognosis of $\mathrm{FHC}$ patients.

\section{Methods}

Protein preparation. Cardiac V3 myosin was prepared from an autopsied human left ventricular muscle as described previously (14). Cardiac heavy meromyosin (HMM) and myosin subfragment 1 (S1) were prepared by $\alpha$-chymotrypsin digestion (18). Actin was prepared from rabbit skeletal muscle acetone powder (19).

Construction of transfer vectors. Total RNA was prepared from a left ventricular muscle obtained during autopsy of a 44-yr-old patient without clinical and pathological evidence of heart disease (20). With two primers designed based upon the published sequence (21, 22 ), a truncated human $\beta$-cardiac MHC cDNA (3414 bp) encoding Met $^{1}$-Asp ${ }^{1138}$ was obtained by reverse transcriptase-coupled PCR (RT-PCR) (23), generating a stop codon at 3415 bp and flanking
XbaI sites on both ends (Fig. 1). The forward primer was $5^{\prime}$-CGCCTGGCTCTAGAATGGGAGATTCGGAGATG-3', and the reverse primer was $5^{\prime}$-GCTCTCTAGACTAGTCTGAGCGCAGCTTCTCCAC-3'. This PCR product was digested with XbaI and subcloned into pBluescript SK II (+) (Stratagene, La Jolla, CA). The sequence of the obtained cDNA was confirmed to be identical to that published previously by dideoxynucleotide termination method using Sequenase 2.0 (United States Biochemical, Cleveland, OH). Four point mutations, $746^{\mathrm{G} \rightarrow \mathrm{A}}, 1208^{\mathrm{G} \rightarrow \mathrm{A}}, 1357^{\mathrm{C} \rightarrow \mathrm{T}}$, and $1816^{\mathrm{G} \rightarrow \mathrm{A}}$ were introduced into the $\beta$-MHC cDNA individually using a transformer site directed mutagenesis kit (Clontech Inc., Palo Alto, CA). These mutations resulted in substitutions of $\mathrm{Arg}^{249}$ for Gln (Arg249Gln), $\mathrm{Arg}^{403}$ for Gln (Arg403Gln), $\mathrm{Arg}^{453}$ for Cys (Arg453Cys), and $\mathrm{Val}^{606}$ for Met (Val606Met), respectively, which have been shown to be responsible for FHC (6). Wild and mutant human cardiac $\beta$-MHC cDNAs thus prepared were excised with $\mathrm{XbaI}$ digestion, and subcloned into a baculovirus transfer vector, pBlueBacM (Invitrogen, San Diego, CA) using an unique NheI site.

A full length cDNA encoding the human ventricular light chain 1 (LC1) (24) was a generous gift from Dr. Yazaki (University of Tokyo, Tokyo, Japan). The cDNA was excised from a eukaryotic expression

pBluescript SK II (+) digested with $\mathrm{Xbal}$

Human left ventricular total RNA

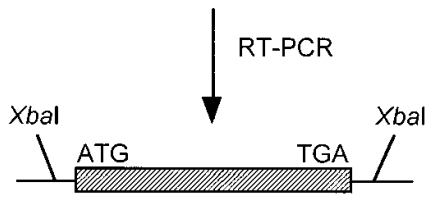

Human $\beta-M H C$ cDNA
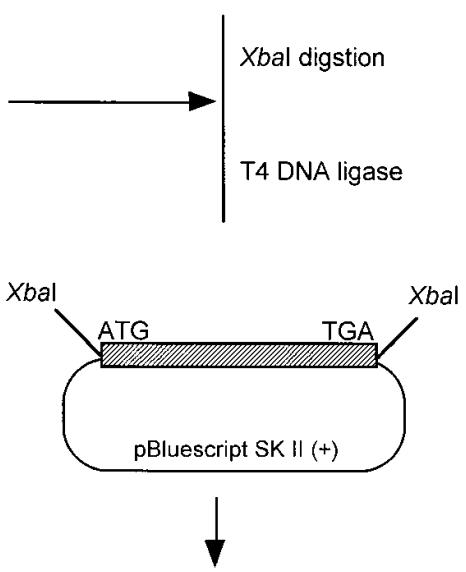

site directed mutagenesis

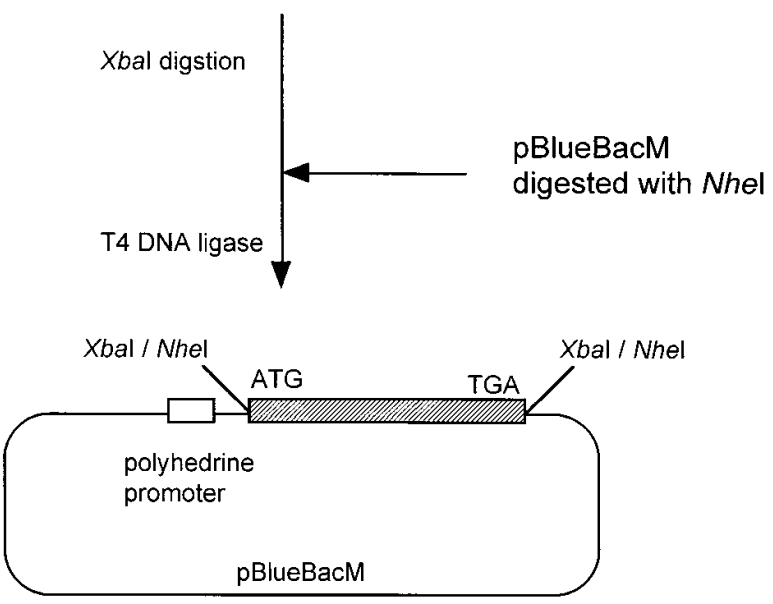

Figure 1. Construction of the human $\beta-\mathrm{MHC}$ cDNA transfer vector. A detailed strategy for constructing the transfer vector is described in Methods. The first 3414 bp of the 5 '-terminus of human $\beta$-MHC cDNA were synthesized by RT-PCR from human left ventricular total RNA. The cDNA fragment is represented as a shaded box. ATG and TAG indicate initiation and termination codons, respectively. The PCR product was excised with $\mathrm{XbaI}$ digestion and subcloned into pBluescript SK II (+). After introduction of a missense mutation, the cDNA was excised with $\mathrm{XbaI}$ digestion and subcloned into a baculovirus transfer vector, pBlueBacM. 
vector, pDR540 (Pharmacia LKB Biotechnology Inc., Uppsala, Sweden), with BamHI digestion and subcloned into pBlueBacM using a BamHI site. A full length cDNA encoding the human ventricular light chain 2 (LC2) was obtained from human left ventricular total RNA by RT-PCR with primers designed based upon the published sequence (25), generating flanking XbaI sites on both ends. The forward primer was 5'-CGCATCTAGAATGGCACCTAAGAAAGCAAAG-3'. The reverse primer was 5'-GACTTCTAGACTAGTCCTTCTCTTCTCCG-3'. This PCR product was digested with XbaI and subcloned into pBlueBacM using a NheI site.

Purification of the expressed human cardiac myosin. Recombinant baculovirus was obtained for each cDNA construct by the protocols described by O'Reilly et al. (26). To express a truncated human ventricular myosin fragment, Sf21 cells were coinfected with the recombinant viruses expressing truncated $\beta-\mathrm{MHC}, \mathrm{LC} 1$, and LC2 $(11,27)$. The cells were harvested $84 \mathrm{~h}$ after infection. The expressed human cardiac myosin was purified as described previously (27).

Gel electrophoresis, immunoblot, and ATPase assay. SDS polyacrylamide gel electrophoresis was carried out on a $7.5-20 \%$ polyacrylamide gradient slab gel. Immunoblots were performed as described previously (28) using an anti-human $\beta$-MHC monoclonal antibody (HMC14) and an anti-human ventricular LC1 antibody
(MLM544) (generous gifts from Dr. Katoh, Yamasa Corp., Chiba, Japan). Nondenaturing gel electrophoresis was performed according to Persechini et al. (29) in the presence of $2 \mathrm{mM} \mathrm{Mg}^{2+}$-ATP. ATPase activity was measured as described previously (30) under conditions described in the legend to Table I.

In vitro motility assay. The in vitro motility assay was performed as described previously (14). Each video frame was digitized at a rate of five frames/s into a $320 \times 240$ pixel array by a video grabber card (Video Blaster; Creative Labs, Milpitas, CA) installed in a personal computer (Compudyne 614295; CompUSA, Dallas, TX). Actin filament velocity was calculated from the movement distance and the elapsed time in successive snapshots.

Statistical analysis. For each myosin, measurements were made on three different preparations expressed independently. All data are presented as mean value \pm SD. Student's $t$ test was used for statistical comparison of mean values. A value of $P<0.05$ was considered to be significant.

\section{Results}

Expression and purification of human $\beta$ cardiac myosin. The produced human $\beta$-MHC contains $\mathrm{Met}^{1}-\mathrm{Asp}^{1138}$ with calcu-

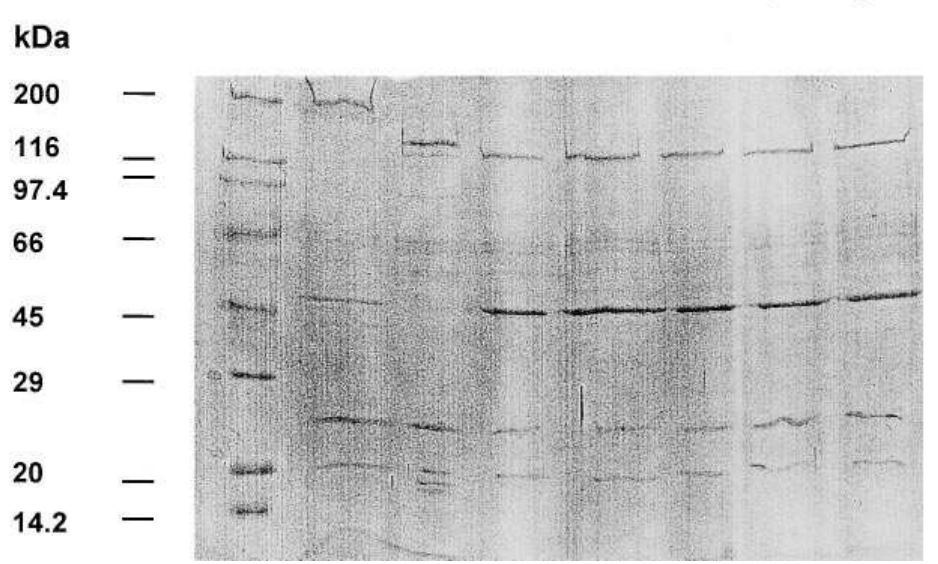

B

1

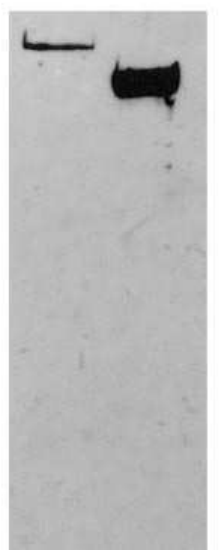

$\mathrm{kDa}$

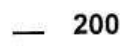

- 116

-

$-66$

$-45$

$-29$

$-20$

14.2
1

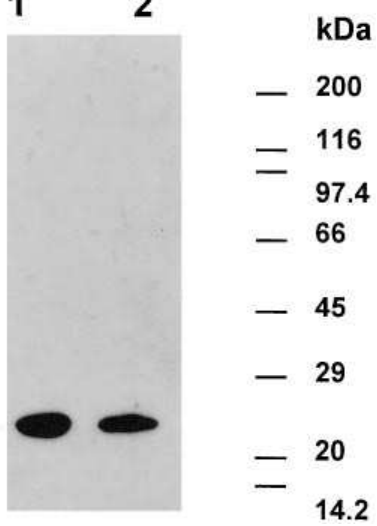

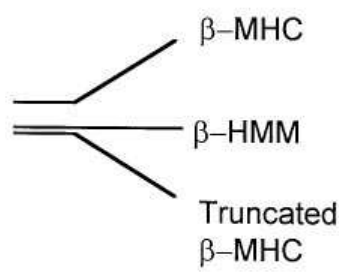

$\longrightarrow$ LC1

LC2
Figure 2. Isolation of the human $\beta$ cardiac myosin expressed in insect cells. (A) $7.5-12 \%$ SDS-polyacrylamide gel electrophoresis of myosin fragments. (lane 1) Molecular weight standard; (lane 2) naturally isolated human cardiac myosin; (lane 3) human cardiac HMM; (lane 4) truncated human cardiac myosin (wild-type) purified from insect cells; (lane 5) Arg249Gln mutant myosin; (lane 6) Arg403Gln mutant myosin; (lane 7) Arg453Cys mutant myosin; (lane 8) Val606Met mutant myosin. (B) Immunoblot of human cardiac myosins (wildtype) using a monoclonal antibody, HMC14, which recognizes human $\beta$-MHC. (lane 1) naturally isolated human cardiac myosin; (lane 2) expressed human cardiac myosin. (C) Immunoblot of human cardiac myosins (wild-type) using a monoclonal antibody, MLM544, which recognizes human cardiac LC1. (lane 1) Naturally isolated human cardiac myosin; (lane 2) expressed human cardiac myosin. 
lated molecular mass of $130 \mathrm{kD}$ thus containing the entire S1 and most of the $\mathrm{S} 2$ portions of myosin. We chose to produce the heavy chain construct containing both the $\mathrm{S} 1$ and $\mathrm{S} 2$ portions of the molecule, because the $\mathrm{S} 2$ segment is necessary for a stable two-headed structure which is important to represent native motor activity of myosin (27) and because it is technically difficult to express an entire $\beta$-MHC (1,935 amino acids) in insect cells. After purification, both wild and mutant myosins contained a 130-kD apparent molecular mass heavy chain, a 25-kD LC1, and a 19-kD LC2 (Fig. $2 A$ ). Approximate stoichiometry of the three polypeptides was 1:1:1 for both wild and mutant $\beta$-MHCs based upon gel densitometry, indicating that the recombinant myosins consisted of one heavy chain and one of each class of light chains as is found in naturally isolated cardiac V3 myosin. For both wild-type and mutant myosins, $\sim 200 \mu \mathrm{g}$ of the purified myosins were obtained from 10 grams of Sf 21 cells.

To confirm the authenticity of the expressed proteins, immunoblot analyses were performed using two kinds of antibodies recognizing either human $\beta$-myosin heavy chain (HMC14) or human ventricular LC1 (MLM544) (31). The former antibody recognized the expressed truncated $\beta$-MHC as well as a naturally isolated human ventricular MHC (Fig. $2 \mathrm{~B}$ ). The latter antibody reacted with both the expressed LC1 and the naturally isolated one (Fig. $2 C$ ) suggesting that the expressed myosin fragment is the human cardiac $\beta$-myosin.

Nondenaturing gel electrophoresis was used to determine whether the expressed $\beta$-MHC forms a double-headed structure or a single-headed structure. As shown in Fig. 3, the entire fraction of the expressed myosin migrated at the similar position to that of the naturally isolated cardiac heavy meromyosin (HMM) but much slower than naturally isolated cardiac S1, suggesting that the expressed $\beta$-MHC forms a stable dimer, i.e., $\mathrm{V} 3$ isoform.

ATPase activities of the expressed myosin. The enzymatic function of the expressed myosins was evaluated by measuring $\mathrm{K}^{+}$EDTA-activated and $\mathrm{Ca}^{2+}$-activated ATPase activities as well as the actin activated $\mathrm{Mg}^{2+}$-ATPase activity. The obtained values for the wild-type recombinant $\beta$-myosin ATPase are shown in Table I. These values are similar to those measured for naturally isolated V3 cardiac HMM (32) or S1 (33) indicating that the expressed $\beta$-myosin is properly folded and retains native enzymatic properties. While the $\mathrm{K}^{+}$EDTA-ATPase activity as well as $\mathrm{Ca}^{2+}$-ATPase activity of the Arg403Gln and the Val606Met mutant myosins was not significantly

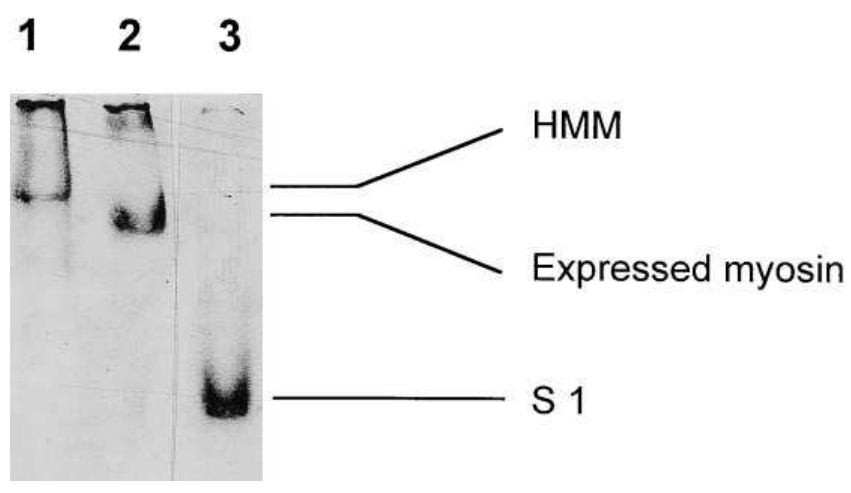

Figure 3. Nondenaturing gel electrophoresis of cardiac myosin fragments. (lane 1) Human cardiac HMM; (lane 2) truncated cardiac myosin expressed in insect cells (wild-type); (lane 3) human cardiac myosin S1. different from those of the wild-type myosin, the Arg249GIn and Arg453Cys mutants showed a large difference. The Arg249Gln mutant showed a decreased $\mathrm{Ca}^{2+}$-ATPase activity without significant change in $\mathrm{K}^{+}$EDTA-ATPase activity, while the Arg453Cys mutation resulted in significant decrease in both $\mathrm{K}^{+}$EDTA- and $\mathrm{Ca}^{2+}$-ATPase activities, suggesting that these mutations change the conformation of the ATPase active site.

Consistent with these results, the $V_{\max }$ of actin-activated ATPase activity was significantly decreased for both Arg249GIn and Arg453Cys mutants without changing the apparent dissociation constant for actin $\left(K_{\mathrm{a}}\right)$. On the other hand, while both $\mathrm{Ca}^{2+}$ - and $\mathrm{K}^{+}$EDTA-ATPase activities were unchanged, the Arg403Gln mutant showed a significantly decreased $V_{\text {max }}$ (31\% of the wild-type) and a significantly increased $K_{\mathrm{a}}(3.3$ times higher than that of the wild-type) for the actin activated ATPase activity suggesting the change in the actin dependent process of myosin ATPase reaction rather than the direct effects on the ATPase active site. The ATPase activities of the Val606Met mutant were not significantly different from those of the wild-type myosin.

Actin-translocating activity of the expressed myosin fragments. Fig. 4 shows the sliding velocity of actin filaments on the recombinant human cardiac myosins. More than $90 \%$ of actin filaments moved smoothly and unidirectionally on all myosins. There was no significant difference between the wild-type and the mutant myosins in the proportion of the moving actin filaments. The sliding velocity for the wild-type myosin was $1.64 \pm 0.14 \mu \mathrm{m} / \mathrm{s}$ at $30^{\circ} \mathrm{C}$. This value is similar to that of naturally isolated V3 cardiac myosin (14-16), further confirming the authenticity of the expressed myosin. The sliding veloci-

Table I. ATPase Activities of the Expressed Human Cardiac Myosins

\begin{tabular}{|c|c|c|c|c|}
\hline & \multirow[b]{2}{*}{$\begin{array}{c}\mathrm{K}^{+} \text {EDTA } \\
\text { ATPase* }\end{array}$} & \multirow[b]{2}{*}{$\begin{array}{c}\mathrm{Ca}^{2+} \\
\text { ATPase }\end{array}$} & \multicolumn{2}{|c|}{$\begin{array}{l}\text { Actin-activated } \\
\text { ATPase activity }\end{array}$} \\
\hline & & & $\operatorname{Vmax}\left(\mathrm{s}^{-1}\right)$ & $K_{\mathrm{a}}$ \\
\hline & $\begin{array}{l}\text { nmol Pi/mg } \\
\text { per min }\end{array}$ & $\begin{array}{l}\text { nmol Pi/mg } \\
\text { per min }\end{array}$ & & $(\mu M)$ \\
\hline Wild type & $198 \pm 15$ & $480 \pm 34$ & $3.31 \pm 0.30$ & $30 \pm 4$ \\
\hline Arg249Gln & $163 \pm 35$ & $111 \pm 17 \|$ & $1.88 \pm 0.23 \|$ & $33 \pm 5$ \\
\hline Arg403Gln & $187 \pm 11$ & $477 \pm 5$ & $1.01 \pm 0.15^{\|}$ & $98 \pm 7 \|$ \\
\hline Arg453Cys & $74 \pm 13 \|$ & $161 \pm 8^{\|}$ & $0.79 \pm 0.11^{\|}$ & $38 \pm 5$ \\
\hline Val606Met & $168 \pm 33$ & $451 \pm 11$ & $2.98 \pm 0.39$ & $41 \pm 6$ \\
\hline
\end{tabular}

Measurements were made on three independent preparations of each myosin. All data are presented as mean activity \pm S.D. $* \mathrm{~K}^{+}$EDTA-ATPase activity was measured in $0.01 \mathrm{mg} / \mathrm{ml}$ myosin, $0.1 \mathrm{mM}$ ATP, $10 \mathrm{mM}$ EDTA, $50 \mathrm{mM}$ Tris- $\mathrm{HCl}(\mathrm{pH} 7.5), 0.6 \mathrm{M} \mathrm{KCl}$ at $25^{\circ} \mathrm{C}$. The reaction was started by adding ATP and the liberated inorganic phosphate was measured as described previously (30). ${ }^{\ddagger} \mathrm{Ca}^{2+}$-ATPase activity was measured in $0.01 \mathrm{mg} / \mathrm{ml}$ myosin, $0.1 \mathrm{mM}$ ATP, $2 \mathrm{mM} \mathrm{CaCl}_{2}, 50 \mathrm{mM}$ Tris- $\mathrm{HCl}(\mathrm{pH}$ 7.5), and $0.04 \mathrm{M} \mathrm{KCl}$ at $25^{\circ} \mathrm{C} .{ }^{8}$ Actin-activated ATPase activity was measured at $25^{\circ} \mathrm{C}$ in $0.01 \mathrm{mg} / \mathrm{ml}$ myosin, $0.1 \mathrm{mM} \mathrm{ATP}, 50 \mathrm{mM} \mathrm{KCl}, 20 \mathrm{mM}$ imidazole- $\mathrm{HCl}(\mathrm{pH} 7.5), 6 \mathrm{mM} \mathrm{MgCl}_{2}, 0.5 \mathrm{mM}$ EGTA with various concentrations of F-actin $(0.1-3.2 \mathrm{mg} / \mathrm{ml})$. The maximum hydrolysis rate $\left(V_{\max }\right)$ and the apparent dissociation constant for actin $\left(K_{\mathrm{a}}\right)$ for each preparation were determined by using a computed nonlinear leastsquare curve-fitting program based on a equation, $V=V_{\max } /\left(1+K_{\mathrm{a}} /[\right.$ actin] $)$ (42). $\| P<0.05$ versus wild type. 


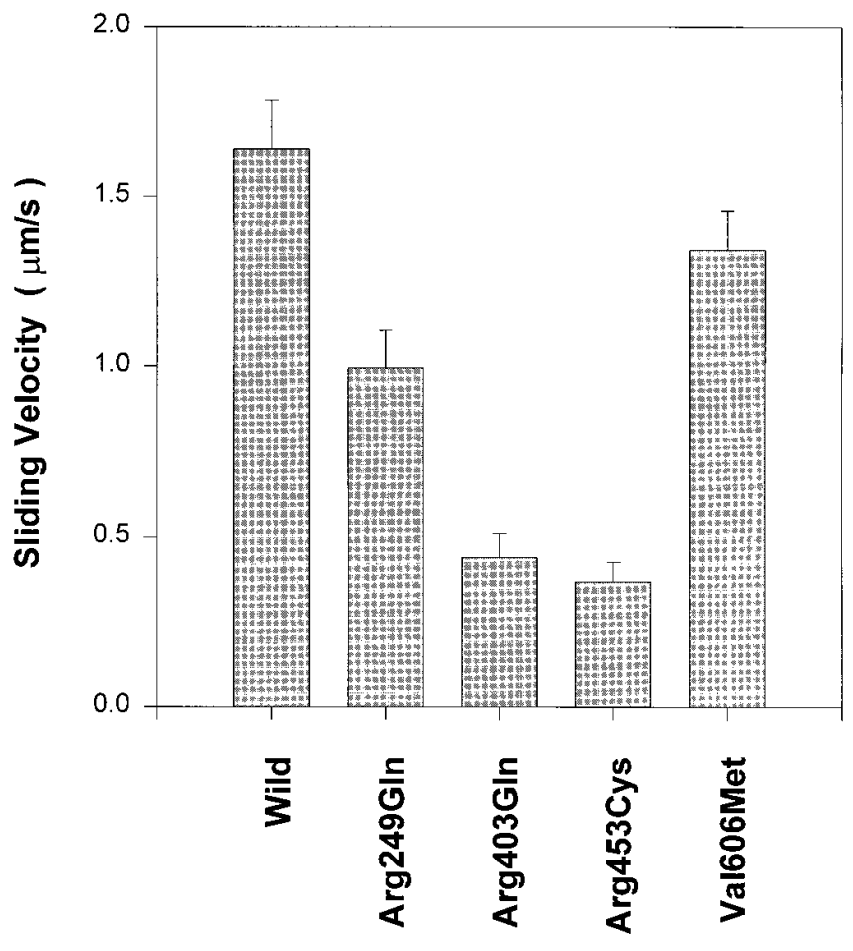

Figure 4. Sliding velocity of actin filaments on the expressed myosins. Actin movement was observed in $30 \mathrm{mM} \mathrm{KCl}, 5 \mathrm{mM} \mathrm{MgCl}, 25 \mathrm{mM}$ imidazole-HCl (pH 7.5), 1 mM EGTA, 1\% 2-mercaptoethanol, 0.5\% methylcellulose, $4.5 \mathrm{mg} / \mathrm{ml}$ glucose, $216 \mu \mathrm{g} / \mathrm{ml}$ glucose oxidase, 36 $\mu \mathrm{g} / \mathrm{ml}$ catalase, and $2 \mathrm{mM}$ ATP at $30^{\circ} \mathrm{C}$. Measurements were made on three independent preparations of each myosin. 20-30 actin filaments were measured to obtain an average velocity for each preparation. All values are mean velocities \pm SD.

ties for the Arg249Gln, Arg403Gln, Arg453Cys, and Val606Met mutants were $1.00 \pm 0.11,0.44 \pm 0.07,0.37 \pm 0.06$, and $1.34 \pm 0.11$ $\mathrm{mm} / \mathrm{s}$, respectively. All of the mutations resulted in significant decrease in the actin translocating activity $(P<0.05)$. The degree of the decrease in the actin translocating activity was correlated with the decrease in $V_{\max }$ of the actin activated ATPase activity. Particularly, the Arg403Gln and the Arg453Cys mutations most drastically decreased the velocity by 73 and $77 \%$, respectively.

\section{Discussion}

In this study, we succeeded in functionally expressing human cardiac $\beta$-myosins in insect cells (Fig. $5 A$ ). Wild-type myosin was well purified with similar enzymatic and motor activities to those of the naturally isolated V3 myosin (14-16, 32, 33), indicating that the recombinant human cardiac myosin mutants can be used to evaluate the motor activities of the myosin expressed in patients' hearts. Another concern for the use of recombinant proteins is that mutation might change overall protein folding and it was reported that substitutions of two amino acid residues in smooth muscle myosin heavy chain resulted in a sixfold decrease in isolatable amount of the expressed myo$\sin (34)$. In this study, however, all of four mutant myosin fragments were well purified with similar yields to that for the wild-type, indicating that these point mutations do not affect proper folding of myosin.

All of four point mutations resulted in decrease in myosin motor function in terms of ATPase and unloaded actin trans- locating activities. While the force generating capacity of the recombinant myosins has not been measured, it is plausible that the power production of the mutant myosin is reduced since the ATP consumption rate of actomyosin is considerably decreased by the mutations. Of importance is the finding that the degree of the dysfunction by the mutation is closely correlated with the prognosis of the disease. According to Watkins et al. (6), cumulative survival rates at 50-yr-old for Arg249Gln, Arg403Gln, Arg453Cys, and Val606Met are 79, 36, 34, 94\%, respectively. This coincides well with the extent of the decrease in myosin motor activity by the mutation. Arg403Gln and Arg453Cys, which decrease enzymatic and motor activities severely (70-80\% inhibition), are associated with poor prognosis. The Arg249Gln mutation, whose effects on myosin motor function are intermediate (40-50\% inhibition), is associated with intermediate prognosis. The Val606Met mutation, which affects myosin function mildly (10-15\% inhibition), is associated with good prognosis. The results suggest that myosin dysfunction caused by the point mutations is responsible for the pathogenesis of FHC, although the relative expression level of mutant myosin in patient's heart might also contribute to the pathogenesis since the expression of the mutant myosin is heterogeneous where the percent mutant expression is unknown from individual to individual. Furthermore, the results of present study imply that the functional analysis of a certain mutation observed in FHC could predict the clinical outcome of the kindreds carrying such a mutation, whose phenotype has not been clinically studied extensively.

The present results also provide fundamentally important information for understanding the structure/function relationship of myosin. The mutation of Arg249Gln or Arg453Cys not only resulted in the decrease in actin translocating activity but also accompanied with a significant reduction of $\mathrm{Ca}^{2+}$-ATPase activity. While the Arg249Gln mutation markedly decreased $\mathrm{Ca}^{2+}$-ATPase activity, the inhibition of $\mathrm{K}^{+}$-ATPase activity was moderate. This might suggest that $\mathrm{Arg}^{249}$ influences the coordination of the metal ion of the ATP substrate to myosin molecule. According to the three-dimensional structure of skeletal muscle myosin S1 (35-37), $\mathrm{Arg}^{249}$ (corresponding to $\mathrm{Arg}^{251}$ of skeletal myosin) is in the $\beta$-strand six located at the outer end of the nucleotide binding pocket (Fig. $5 \mathrm{C}$ ). This residue is in close proximity of $\mathrm{Asp}^{461}\left(11.6 \AA\right.$ between $\mathrm{Arg}^{251}$ and $\mathrm{Asp}^{463}$ in skeletal myosin) which participates in the coordination of the $\mathrm{Mg}^{2+}$ ion of the ATP substrate $(35,38,39)$ which is consistent with the observed change in myosin function. On the other hand, the Arg453Cys mutation decreased both $\mathrm{Ca}^{2+}$ and $\mathrm{K}^{+}$-EDTA ATPase activities to similar extent, suggesting that the mutation influences either ATP binding or the catalysis of ATP hydrolysis. Arg ${ }^{453}$ is located at the end of the nucleotide binding pocket (37). Although this residue is a little distal from the residues participated in $\gamma$-phosphate binding $(\sim 20 \AA)$, it resides in a loop next to the $\alpha$-helix containing $\mathrm{Asp}^{461}, \mathrm{Ala}^{463}, \mathrm{Gly}^{464}$, and $\mathrm{Glu}^{466}$ (corresponding to $\mathrm{Asp}^{454}, \mathrm{Ser}^{456}$, $\mathrm{Gly}^{457}$, and $\mathrm{Glu}^{459}$ of Dictyostelium discoideum myosin) which are suggested to participate in the binding of $\gamma$-phosphate of $\operatorname{ATP}(38,39)$. One of the residues close to $\mathrm{Arg}^{453}$ is $\operatorname{Glu}^{448}(10.8$ $\AA$ between $\mathrm{Arg}^{455}$ and $\mathrm{Asp}^{450}$ in skeletal myosin) and it would be plausible that charge interaction between $\mathrm{Arg}^{453}$ and an acidic residue contributes to position the $\alpha$-helix containing the several residues involved in $\gamma$-phosphate binding.

Different from above mutations, the Arg403Gln mutation did not change the $\mathrm{Ca}^{2+}$ - and $\mathrm{K}^{+}$-EDTA ATPase activities significantly but only decreased the actin dependent ATPase ac- 


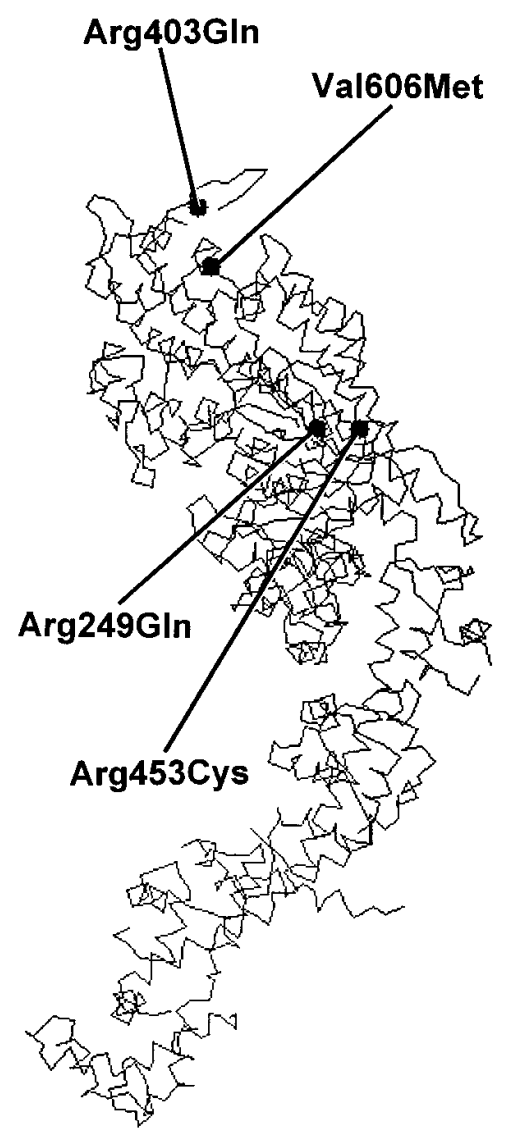

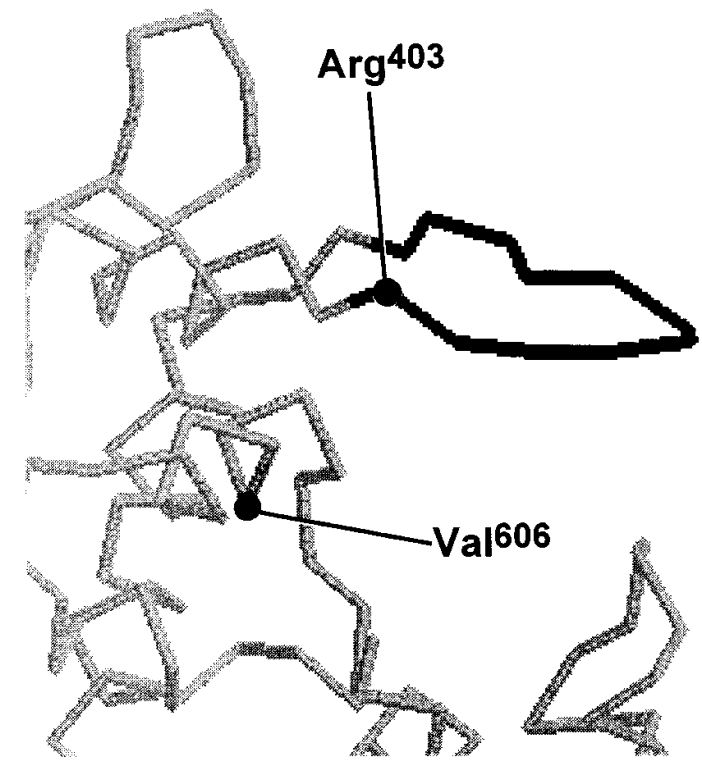

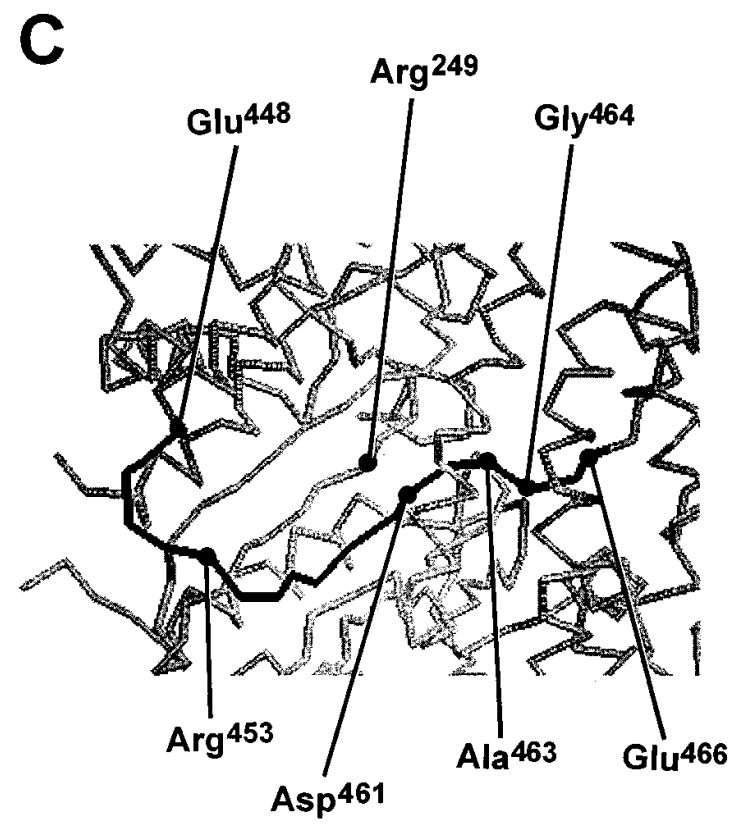

Figure 5. Locations of the point mutations expressed in this study. $(A)$ The three-dimensional structure of the chicken skeletal myosin S1 (35) showing the positions equivalent to the point mutations in $\beta$ cardiac myosin; $(B)$ a close-up stereoview of the region of the actin binding interface. The $\mathrm{Arg}^{403}$ - $\mathrm{Lys}^{413}$ loop is indicated in a bold line; $(C)$ a close-up stereoview of the ATP $\gamma$-phosphate binding pocket. The loop containing Glu ${ }^{448}$, $\mathrm{Arg}^{453}, \mathrm{Asp}^{461}, \mathrm{Gly}^{464}$, and $\mathrm{Glu}^{466}$ is indicated in a bold line. tivity. The result suggests that this mutation changes the ATPase reaction steps involved in the actin dependent process (40). Consistent with this finding, the apparent dissociation constant $\left(K_{\mathrm{a}}\right)$ was significantly increased for Arg403Gln mutant. The observed functional change is well explained by structural point of view that $\mathrm{Arg}^{403}$ lies in the loop ( $\mathrm{Arg}^{403}$ $\mathrm{Lys}^{413}$ ) which forms a close contact with actin $\left(\mathrm{Pro}^{332}\right.$ to $\mathrm{Glu}^{334}$ of actin molecule) (Fig. 5 B) (36). The Arg403Gln mutation greatly decreased the actin translocating activity suggesting the importance of this loop for motor activity of myosin.

$\mathrm{Val}^{606}$ is in a helix-loop-helix motif just at the upstream of the junction of 50-20 kD subdomains of S1 which contain a part of actin binding interface of myosin molecule $(35,37)$. The effect of Val606Met mutation on myosin motor and enzymatic activities was mild. On the basis of the structural analysis of acto-S1 complex (36), there are no indications of the importance of this segment for actin-myosin interaction, therefore, the very mild modulation of myosin function by the Val606Met mutation would be reasonable, although the mild functional modulation would be partly due to the conserved substitution of the amino acid residue, i.e., Val to Met.

It is still unclear how a missense mutation leads to cardiac hypertrophy, myocardial fiber disarray, or fatal arrhythmia, although it may be plausible that myosin motor dysfunction constitutes the primary stimulus for the cardiac hypertrophy as compensation for the reduced contractile function resulting 
from the dysfunction of the individual cross-bridge. Although the four mutations tested in this study were different in the degree of the dysfunction of myosin motor, the patients carrying the mutations on the $\beta$-MHC tend to produce cardiac hypertrophy to a similar extent of each other $(1,2,6-9)$, suggesting that cardiac hypertrophy is not directly related to the prognosis of the disease. Consistent with this notion, it was reported that homozygous transgenic mice carrying the Arg403Gln mutation were live-born and had normal gross cardiac anatomy but uniformly died by day 7 (41). Myocardial hypertrophy itself may not be the cause of death but deterioration of cardiac myosin motor function may trigger some process to threaten a life.

In summary, this is the first report which analyzed the effects of point mutations on the motor function of human $\beta$ cardiac myosin in detail. The methodology used in this study enables us to study the structure/function relationship of myosin, providing clinical implication for the prognosis of the patients carrying a certain mutation in the $\beta$-MHC gene.

\section{Acknowledgments}

We thank Dr. Steven A. Fisher (Case Western Reserve University, Cleveland, $\mathrm{OH}$ ) for his valuable comments on this project. We also thank Dr. Yoshio Yazaki and Dr. Masahiko Kurabayashi (University of Tokyo, Tokyo, Japan) for the generous gift of the human ventricular alkali light chain cDNA. We are grateful to Dr. Christine Moravec (Cleveland Clinic Foundation, Cleveland, $\mathrm{OH}$ ) for providing a human ventricular tissue, and to Dr. Hirohisa Katoh (Yamasa Corp., Chiba, Japan) for providing monoclonal antibodies against human $\beta$ myosin heavy chain and ventricular light chain.

This work was supported by Grants HL56218, HL47530 and AR41653, from the National Institutes of Health. M. Sata was supported by research fellowships from the American Heart Association, Massachusetts Affiliate, Inc., the Fukuda Memorial Foundation, and the Ueda Memorial Trust Fund for Research of Heart Diseases.

\section{References}

1. Maron, B.J., R.O. Bonow, R.O. Cannon III, M.B. Leon, and S.E. Epstein. 1987. Hypertrophic cardiomyopathy: interrelations of clinical manifestations, pathophysiology, and therapy. N. Engl. J. Med. 316:780-789, 844-852.

2. Clark, C.E., W.L. Henry, and S.E. Epstein. 1973. Familial prevalence and genetic transmission of idiopathic hypertrophic subaortic stenosis. N. Engl. J. Med. 289:709-714.

3. Geisterfer-Lawiance, A.A.T., S. Kass, G. Tanigawa, H.P. Vosberg, W. McKenna, C.E. Seidman, and J.G. Seidman. 1990. A molecular basis for familial hypertrophic cardiomyopathy: a $\beta$-cardiac myosin heavy chain gene missense mutation. Cell. 12:999-1006.

4. Schwartz, K., L. Carrier, P. Guicheney, and M. Komajda. 1995. Molecular basis of familial cardiomyopathy. Circulation. 91:532-540.

5. Marian, A.J., and R. Roberts. 1995. Recent advances in the molecular genetics of hypertrophic cardiomyopathy. Circulation. 92:1336-1347.

6. Watkins, H., T. Rosenzweig, D.S. Hwang, T. Levi, W. McKenna, C.E. Seidman, and J.G. Seidman. 1992. Characteristic and prognostic implications of myosin missense mutations in familial hypertrophic cardiomyopathy. $N$. Engl. J. Med. 326:1106-1114.

7. Epstein, N.D., G.M. Cohn, F. Cyran, and L. Fananapazir. 1992. Difference in clinical expression of hypertrophic cardiomyopathy associated with two distinct mutations in the $\beta$-myosin heavy chain gene: a 908Leu-Val mutation and a 403 Arg-Gln mutation. Circulation. 86:345-352.

8. Anan, R., G. Greve, L. Thierfelder, H. Watkins, W.J. McKenna, S. Solomon, C. Vecchio, H. Shono, S. Nakao, H. Tanaka, et al. 1994. Prognostic implications of novel $\beta$ myosin heavy chain gene mutations that cause familial hypertrophic cardiomyopathy. J. Clin. Invest. 93:280-285.

9. Fananapazir, L., and N.D. Epstein. 1994. Genotype-phenotype correlations in hypertrophic cardiomyopathy: insights provided by comparisons of kindreds with distinct and identical $\beta$-myosin heavy chain mutations. Circulation. 89:22-32.

10. Cuda, G., L. Fananapazr, W.S. Zhu, J.R. Sellers, and N.D. Epstein. 1993. Skeletal muscle expression and function of $\beta$-myosin in hypertrophic cardiomyopathy. J. Clin. Invest. 91:2861-2865.
11. Sweeney, H.L., A.J. Straceski, L.A. Leinwand, B.A. Tikunov, and L. Faust. 1994. Heterologous expression of a cardiomyopathic myosin that is defective in its actin interaction. J. Biol. Chem. 269:1603-1605.

12. Kurabayashi, M., H. Tsuchimochi, I. Komuro, F. Takaku, and Y. Yazaki. 1988. Molecular cloning and characterization of human cardiac $\alpha$ - and $\beta$ - form myosin heavy chain complementary DNA clones. J. Clin. Invest. 82:524-531.

13. Schwartz, K., A.M. Lompre, P. Bouveret, C. Wisnewsky, and R.G. Whalen. 1982. Comparison of rat cardiac myosin at fetal stages in young animals and in hypothyroid adults. J. Biol. Chem. 193:14412-14418.

14. Sata, M., S. Sugiura, H. Yamashita, S. Momomura, and T. Seizawa. 1993. Dynamic interaction between cardiac myosin isoforms modifies velocity of actomyosin sliding in vitro. Circ. Res. 73:696-704.

15. Harris, D.E., S.S. Works, R.K. Wright, N.R. Alpert, and D.M. Warshaw. 1994. Smooth, cardiac and skeletal muscle myosin force and motor generation assessed by cross-bridge mechanical interaction in vitro. J. Muscle Res. Cell Motil. 15:11-19.

16. VanBuren, P., D.E. Harris, N.R. Alpert, and D.M. Warshaw. 1995. Cardiac V1 and V3 myosins differ in their hydrolytic and mechanical activities in vitro. Circ. Res. 77:439-444.

17. McNally, E.M., R. Kraft, M. Bravo-Zehnder, D.A. Taylor, and L.A. Leinwand. 1989. Full-length rat alpha and beta cardiac myosin heavy chain sequences: Comparisons suggest a molecular basis for functional difference. $J$. Mol. Biol. 210:665-670.

18. Weeds, W.G., and R.S. Taylor. 1975. Separation of subfragment-1 isoenzymes from rabbit skeletal muscle myosin. Nature (Lond.). 257:54-56.

19. Spudich, J.A., and S. Watt. 1971. The regulation of rabbit skeletal muscle contraction. J. Biol. Chem. 246:4866-4871.

20. Chomczynski, P., and N. Sacchi. 1987. Single-step method of RNA isolation by acid guanidinium thiocyanate-phenol-chloroform extraction. Anal. Biochem. 162:156-169.

21. Jaenicke, T., K.W. Diederich, W. Haas, J. Schleich, P. Lichter, M. Pfordt, A. Bach, and H.P. Vosberg. 1990. The complete sequence of the human $\beta$-myosin heavy chain gene and a comparative analysis of its product. Genomics. 8:194-206.

22. Liew, C.C., M.J. Sole, K. Yamauchi-Takihara, B. Kellam, D.H. Anderson, L. Lin, and J.C. Liew. 1990. Complete sequence and organization of the human cardiac $\beta$-myosin heavy chain gene. Nucleic Acids Res. 18:3647-3651.

23. Kelley, C.A., M. Takahashi, J.H. Yu, and R.S. Adelstein. 1993. An insert of seven amino acids confers functional differences between smooth muscle myosins from the intestines and vasculature. J. Biol. Chem. 268:12848-12854.

24. Kurabayashi, M., I. Komuro, H. Tsuchimochi, F. Takaku, and Y. Yazaki. 1988. Molecular cloning and characterization of human atrial and ventricular myosin alkali light chain cDNA clones. J. Biol. Chem. 263:13930-13936.

25. Libera, I.D., E. Hoffmann, M. Eloroff, and G. Jackowski. 1989. Isolation and nucleotide sequence of the cDNA encoding human ventricular myosin light chain 2. Nucleic Acids Res. 17:2360.

26. O'Reilly, D.R., L.K. Miller, and V.A. Luckow. 1992. Baculovirus expression vectors: A Laboratory Manual, W.H. Freeman and Co., New York. 347 pp.

27. Sata, M., M. Matsuura, and M. Ikebe. 1996. Characterization of the motor and enzymatic properties of smooth muscle long S1 and short HMM: role of the two headed structure on the activity and regulation of myosin motor. Biochemistry. 35:11113-11118.

28. Higashihara, M., L.L. Young-Frada, R. Craig, and M. Ikebe. 1989. Inhibition of conformational change in smooth muscle myosin by a monoclonal antibody against the 17-kDa light chain. J. Biol. Chem. 264:5218-5225.

29. Persechini, A., K.E. Kamm, and J.T. Stull. 1986. Different phosphorylated forms of myosin in contracting trachial smooth muscle. J. Biol. Chem. 261: 6293-6299.

30. Ikebe, M., and D.J. Hartshorne. 1985. Proteolysis of smooth muscle myosin by Staphylococcus aureus protease: preparation of heavy meromyosin and subfragment 1 with intact 20,000 dalton light chains. Biochemistry. 24:2380-2387.

31. Tsuchimochi, H., M. Sugi, M. Kuro-o, S. Ueda, F. Takaku, S. Furuta, T. Shirai, and Y. Yazaki. 1984. Isozymic changes in myosin of human atrial myocardium induced by overload: immunohistochemical study using monoclonal antibodies. J. Clin. Invest. 74:662-665.

32. Margossian, S.S. 1985. Reversible dissociation of dog cardiac myosin regulatory light chain 2 and its influence on ATP hydrolysis. J. Biol. Chem. 260: 13747-13754.

33. Stein, L.A., and M.P. White. 1987. Biochemical kinetics of porcine cardiac subfragment-1. Circ. Res. 60:39-49.

34. Onishi, H., M.F. Morales, K. Katoh, and K. Fujiwara. 1995. The putative actin-binding role of hydrophobic residues $\operatorname{Trp}^{546}$ and $\mathrm{Phe}^{547}$ in chicken gizzard heavy meromyosin. Proc. Natl. Sci. USA. 92:11965-11969.

35. Rayment, I., W.R. Rypniewski, K. Schmidt-Base, R. Smith, D.R. Tomchick, M.M. Benning, D.A. Winkelmann, G. Wesenberg, and H.M. Holden. 1993. Three-dimensional structure of myosin subfragment 1: a molecular motor. Science (Wash. DC). 261:50-58.

36. Rayment, I., H.M. Holden, M. Whittacker, C.B. Yohn, M. Lorenz, K.C. Holmes, and R.A. Milligand. 1993. Structure of the actin-myosin complex and its implications for muscle contraction. Science (Wash. DC). 261:58-65.

37. Rayment, I., H.M. Holden, J.R. Sellers, L. Fananapazir, and N.D. Ep- 
stein. 1995. Structural interpretation of the mutations in the $\beta$-cardiac myosin that have been implicated in familial hypertrophic cardiomyopathy. Proc. Natl. Acad. Sci. USA. 92:3864-3868.

38. Fisher, A.J., C.A. Smith, J.B. Thoden, R. Smith, K. Sutoh, H.M. Holden, and I. Rayment. 1995. X-ray structure of the myosin motor domain of Dictyostelium discoideum complexed with $\mathrm{MgADP} \cdot \mathrm{BeFx}$ and $\mathrm{MgADP} \cdot \mathrm{AlF}_{4}{ }^{-}$. Biochemistry. 34:8960-8972.

39. Smith, C.A., and I. Rayment. 1996. X-ray structure of the Magnesium (II) ADP.Vanadate complex of the Dictyostelium discoideum myosin motor domain to 1.9 Å resolution. Biochemistry. 35:5404-5417.

40. Eisenberg, E., and T.L. Hill. 1985. Muscle contraction and free energy transduction in biological systems. Science (Wash. DC). 227:999-1006

41. Geisterfer-Lowrance, A.A.T., M. Christe, D.A. Conner, J.S. Ingwall, F.J. Schoen, C.E. Seidman, and J.G. Seidman. 1996. A mouse model of familial hypertrophic cardiomyopathy. Science (Wash. DC). 272:731-734.

42. Lutz, R.A., C. Bull, and D. Rodbard. 1986. Computer analysis of enzyme-substrate-inhibitor kinetic data with automatic model selection using IBM-PC compatible microcomputers. Enzyme. 36:197-206. 